\title{
A General Model for Thermal Energy Storage in Combined Heat and Power Dispatch Considering Heat Transfer Constraints
}

\section{DOI:}

10.1109/TSTE.2018.2793360

\section{Document Version}

Accepted author manuscript

Link to publication record in Manchester Research Explorer

Citation for published version (APA):

Dai, Y., Chen, L., Min, Y., Mancarella, P., Chen, Q., Hao, J., Hu, K., \& Xu, F. (2018). A General Model for Thermal Energy Storage in Combined Heat and Power Dispatch Considering Heat Transfer Constraints. IEEE Transactions on Sustainable Energy. https://doi.org/10.1109/TSTE.2018.2793360

\section{Published in:}

IEEE Transactions on Sustainable Energy

\section{Citing this paper}

Please note that where the full-text provided on Manchester Research Explorer is the Author Accepted Manuscript or Proof version this may differ from the final Published version. If citing, it is advised that you check and use the publisher's definitive version.

\section{General rights}

Copyright and moral rights for the publications made accessible in the Research Explorer are retained by the authors and/or other copyright owners and it is a condition of accessing publications that users recognise and abide by the legal requirements associated with these rights.

\section{Takedown policy}

If you believe that this document breaches copyright please refer to the University of Manchester's Takedown Procedures [http://man.ac.uk/04Y6Bo] or contact uml.scholarlycommunications@manchester.ac.uk providing relevant details, so we can investigate your claim.

\section{OPEN ACCESS}




\title{
A General Model for Thermal Energy Storage in Combined Heat and Power Dispatch Considering Heat Transfer Constraints
}

\author{
Yuanhang Dai, Student Member, IEEE, Lei Chen, Member, IEEE, Yong Min, Pierluigi Mancarella, \\ Senior Member, IEEE, Qun Chen, Member, IEEE, Junhong Hao, Kang Hu and Fei Xu
}

\begin{abstract}
Heat transfer (HT) is a major constraint in thermal system analysis. However, when discussing utilizing the flexibility provided by the heating sector, for example using thermal energy storage (TES) to increase operational flexibility of combined heat and power (CHP), the HT process is often ignored. This may mean infeasibility of the resulting schedules. In response to this, this paper proposes a general TES model which takes detailed HT analysis into account. By setting the relevant parameters, this model can be used to describe the HT process for both sensible heat (SH) and latent heat (LH) TES devices. An iteration method is proposed to deal with the nonlinearity introduced by the HT constraints, and to solve the joint nonlinear dispatch problem for CHP with TES. The simulation results show that explicitly considering the HT process is essential to realistically assess and therefore make full use of the flexibility provided by TES. Moreover, the comparison between LH and SH TES shows that LH can provide more flexibility for the system, especially when the starting energy level in the TES device is low.
\end{abstract}

Index Terms-- combined heat and power (CHP), flexibility, heat transfer (HT), power dispatch, thermal energy storage (TES)

\section{NOMENCLATURE}

$A^{g / g-l / l, h s / s t}$

$A^{h s / s t / r l / t e s}$

$c^{m d m / t e s / \text { water }}$

$D / U^{t p / c h p}$

$d^{\text {tes }}$ $k^{g / g-l / h, h s / s t}$

liquid/ liquid sub process for the heat station/ heat storage HT, $\mathrm{m}^{2}$

the HT area of the heat exchanger for heat station/ heat storage/ heat release/ thermal energy storage (TES), $\mathrm{m}^{2}$

specific heat capacity of heat conduction medium/ TES material/ water, $\mathrm{J} /(\mathrm{kg} \cdot \mathrm{K})$

downward/ upward ramping rate of thermal units/ combined heat and power (CHP) units, MW/h

density of TES material, $\mathrm{kg} / \mathrm{m}^{3}$

This work was supported by the National Key R\&D Program of China (2017YFB0902100), the National Natural Science Foundation of China (51377002), the State Grid Corporation of China (SGTJ0000KXJS1500072), the UK-China Joint Research and Innovation Partnership Fund, and the UK EPSRC through the MY-STORE project (EP/N001974/1).

Y. Dai (daiyh13@mails.tsinghua.edu.cn), L. Chen (chenlei08@tsinghua.edu.cn), Y. Min (minyong@tsinghua.edu.cn), J. Hao and F. Xu are with the Department of Electrical Engineering, Tsinghua University, Beijing 100084, China.

P. Mancarella is with the Department of Electrical and Electronic Engineering, The University of Melbourne, Victoria 3010, Australia, and with the School of Electrical and Electronic Engineering, The University of Manchester, M13 9PL, U.K (pierluigi.mancarella@unimelb.edu.au).

Q. Chen and K. Hu are with the Department of Engineering Mechanics, Tsinghua University, Beijing 100084, China. $f^{t p / c h p}(\bullet)$

$F_{i, n}$

$H^{g / g-l /, h s / s t}$

$H^{h s / s t / r l}$

$H^{l s}$

$H^{s t / r l, \max }$

$k$

$k^{h s / s t / r l / t e s}$

$m^{\text {chp }}$

$m^{m d m, s t / r l}$

$m^{\text {pipe }}$

$m^{s t m, h s / s t}$

$m^{s t m, s t, \max }$

$m^{\text {tes,strl }}$

$N$

coal consumption function of thermal units/CHP units, TCE(Ton of Coal Equivalent)

transfer factor of DC power flow from bus $n$ to branch $i$

heat power of gas/ gas-liquid/ liquid sub process for the heat station/ heat storage HT, MW

heat power of heat station/ heat storage/ heat release process, MW

heat loss power of the TES device, MW

maximum heat storage/ release power, MW

iteration number

HT coefficient of gas/ gas-liquid/ liquid sub process for the heat station/ heat storage HT, W/ $\left(\mathrm{m}^{2} \cdot \mathrm{K}\right)$

HT coefficient of the heat exchanger for heat station/ heat storage/ heat release/ TES, $\mathrm{W} /\left(\mathrm{m}^{2} \cdot \mathrm{K}\right)$

steam mass output of CHP, $\mathrm{kg} / \mathrm{s}$

mass flow rate of heat conduction medium for heat storage/ release process, $\mathrm{kg} / \mathrm{s}$

mass flow rate of pipeline water, $\mathrm{kg} / \mathrm{s}$

steam mass flow rate for heat station/ heat storage, $\mathrm{kg} / \mathrm{s}$

maximum steam mass flow rate for heat storage, $\mathrm{kg} / \mathrm{s}$

mass flow rate of TES material for heat storage/ release process, $\mathrm{kg} / \mathrm{s}$

number of total dispatch period

the $k$ th extreme point of electric output/ steam output for $i$ th CHP, MW or kg/s

branch electric power, MW

$P^{b r c h}$

$P^{b r c h, \max / \min }$

maximum/ minimum branch electric power, MW

$P^{c h p / t p / w p}$

$P^{e l}$

$P^{w p, \max }$

$r$

$R^{e n, r l}$

$R^{e n, t e s, s t / r l}$

$R^{\text {tes }}$

$S^{c v, c h p}$

$S^{t p / c h p / e l / b r c h / w p}$ power output of CHP unit/ thermal unit/ wind farm, MW

electric load, MW

available wind power, MW

latent heat of the steam, $\mathrm{MJ} / \mathrm{kg}$

Entransy dissipation-based thermal resistance (EDTR) of the HT process between heat conduction medium and district heating system, K/MW

EDTR of the heat storage/ release process between TES material and heat conduction medium, K/MW thermal resistance for the heat loss process, K/MW set for CHP's extreme points

set for thermal units/ CHP units/ electric loads/ branches/ wind farms

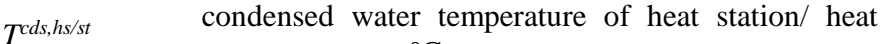
storage process, ${ }^{\circ} \mathrm{C}$

inlet/ intermediate/ output temperature of heat station, ${ }^{\circ} \mathrm{C}$ 


\begin{tabular}{|c|c|}
\hline$T^{h s / s t, 1 / 2}$ & $\begin{array}{l}\text { intermediate temperature of heat station/ heat storage } \\
\text { HT process, }{ }^{\circ} \mathrm{C}\end{array}$ \\
\hline$T^{p h s}$ & phase-change temperature of the extraction steam, ${ }^{\circ} \mathrm{C}$ \\
\hline$T^{\text {st/rl, in/out }}$ & $\begin{array}{l}\text { inlet/ outlet temperature of the heat conduction } \\
\text { medium for heat storage/ release process, }{ }^{\circ} \mathrm{C}\end{array}$ \\
\hline$T^{s t m}$ & extraction steam temperature, ${ }^{\circ} \mathrm{C}$ \\
\hline$T^{t e s}$ & TES material temperature, ${ }^{\circ} \mathrm{C}$ \\
\hline$T^{\text {tes, in/out }}$ & inlet/ outlet temperature of the TES material, ${ }^{\circ} \mathrm{C}$ \\
\hline$T^{t e s, \max / \min }$ & maximum/ minimum TES material temperature, ${ }^{\circ} \mathrm{C}$ \\
\hline$V^{t e s}$ & volume of the TES device, $\mathrm{m}^{3}$ \\
\hline$\gamma$ & updating step of iteration \\
\hline$\delta$ & threshold of iteration \\
\hline$\Delta t$ & time interval of each dispatch period \\
\hline$\eta_{i, k, t}$ & coefficient for CHP's extreme points \\
\hline$\lambda / \mu$ & $\begin{array}{l}\text { coefficient of coal consumption function } f^{p}(\cdot \\
f^{c h p}(\bullet)\end{array}$ \\
\hline$\rho^{h-m, h s / s t}$ & $\begin{array}{l}\text { heat-mass ratio for heat station/ heat storage HT } \\
\text { process, } \mathrm{MJ} / \mathrm{kg}\end{array}$ \\
\hline$\Phi^{t e s}$ & thermal energy stored in the TES device, MWh \\
\hline$\Phi^{t e s, \max / \min }$ & $\begin{array}{l}\text { maximum/ minimum thermal energy of the TE } \\
\text { device, } \mathrm{MWh}\end{array}$ \\
\hline
\end{tabular}

The subscript $i, t$ represents device $i$ at time period $t$.

\section{INTRODUCTION}

$\mathrm{T}^{\circ}$ O accommodate variable renewable energy sources (VRESs), for example wind and solar, as much as we can, it is universally recognized that there needs more flexibility, either from power system itself [1] or from other energy vectors [2][3]. If there is not enough flexibility in the system, it is highly possible that much of the available VRES would need to be curtailed, which is just the case in Northern China. In these Northern provinces where wind resources are abundant, the flexibility in power system is very limited due to the large proportion of combined heat and power (CHP) capacity [4], especially in winter when the wind resource is extremely rich and the heating demand of CHP is high. Generally, the coalfired CHP is inherently less flexible than gas-fired turbines, and the coupling between heat and power further limits the flexibility the CHP can provide [5]. This can be one of the primary reasons for wind curtailment problem, where in some provinces the curtailed wind energy accounts for more than $40 \%$ of the available resources in 2016 [6]. Therefore, to increase the flexibility of CHP can be an effective solution for the wind curtailment problem.

Among all the possible means for improving flexibility for CHP, thermal energy storage (TES) is attracting increasing interest due to its high efficiency, large capacity as well as low cost [7]. Some Northern European countries have been using TES to increase the flexibility for CHP for decades [8]-[12]. Reference [8] proposes a self-supply strategy in Denmark which utilizes TES to help with meeting the heat demand and deal with the fluctuations of the wind generation; the EnergyPLAN model is introduced in [9], in which TES is mainly used to minimize the electricity export in Denmark; different solutions regarding the use of electric heating, heat pump (HP) and TES are compared in [10] to integrate wind power; further Danish experience of design and utilize small CHP with TES in market environment is presented in [11]; Reference [12] summarizes some recent development in Denmark about how CHP equipped with TES and electric boiler can benefit the overall system efficiency and the largescale integration of wind and solar power. Because of the current VRES accommodation issue, TES has become the focus of more recent studies. TES devices [13]-[19], together with electrical heating boilers [13][14][17][19] or/and HPs [13][20] have been co-optimized to increase flexibility of CHP, and thus promoting wind power integration. On the other hand, using the TES capacity of the existing thermal system components like pipelines in the district heating system [19][21]-[24] or the building envelop [20][23][24][25] is also being investigated to provide extra flexibility. To be more specific, the technical and economic characteristics of distributed multi-energy system including technologies such as CHP, TES and HP are analyzed in [13]; the chances of utilizing electric boiler and TES to increase the flexibility of CHP is explored in [14], and a linear model is also proposed for the water based heat storage tank; a detailed phase-change TES device model is proposed in [15] for the integration of renewable power sources; the use of both TES and electric energy storage for the integration of wind power is studied in [16]; solar thermal plant and TES are utilized in [17] for the flexible operation of an CHP based district heating system, in which a model suitable for both planning and operation is proposed; based on long time forecast price and heat demand, a CHP with TES is used to minimize the total cost of the system in [18]; reference [19] proposed an integrated scheme of electric and thermal system to accommodate more variable renewable power by considering $\mathrm{CHP}$, heat pump, heat storage and the constraints of the district heating network; in [20] TES is used to increase the flexibility of CHP, and HP and building thermal inertia are used to make the electric load more flexible so that it can follow the output of wind power and therefore to improve wind accommodation; the TES capacity of the district heat system is exploited in [21] and [22] to increase the flexibility of CHP; the potential of using electric heating boiler with TES device, district heating system as well as building thermal inertia as a flexible load is explored in [23], and reference [24] studies the integrated dispatch concerning these components; reference [25] investigated a feasible region method of deploy the building thermal inertia to reduce wind curtailment. In almost all the above studies, TES is typically modelled with constant heat storage and release power limit, and these two limits are equal to each other.

However, the heat storage and release processes are complex and nonlinear heat transfer (HT) processes entailing the interaction between the hot and cold fluids. For this reason, both the heat storage and release power are functions of the status of the hot and cold fluids as well as of the characteristics of the corresponding heat exchangers. Hence, heat storage/release power limits should not be constant, but rather time-varying and related to the working conditions of the systems. Previous works have only addressed the energy 
balance constraints of the TES device by ignoring the detailed HT process, with constant heat storage and release power limits. Therefore, there would be no difference among different working conditions with such given fixed heat storage and release power limits, and the analysis would be much easier. Under certain circumstances like planning based on multiple scenarios [26][27], this simplification is acceptable and practical since detailed HT model may be too complex and will add to the already existed computation burden. However, the additional flexibility that the TES can actually provide is more directly related to the heat storage/release power instead of the TES capacity. Therefore, in the operational timescale TES needs a more accurate modelling approach in order to adequately assess its effectiveness and limitations in the flexibility enhancement for the CHP dispatch.

There are three kinds of TES, namely, sensible heat (SH), latent heat (LH) and thermo-chemical TES [28]. Though needing more space, SH TES costs relatively less compared to the other two options and is already commercially feasible. There are various LH TES demonstration projects under construction, while the large-scale thermo-chemical TES is still under development. The abovementioned studies are mostly based on SH TES with water as the storage medium. Different TES technologies will naturally have different operation characteristics and inevitably have different influence on the flexibility they can provide as well as system performance. However, in previous studies the difference between SH TES and LH TES only lies in the thermal energy balance equation and there is no difference between the heat storage/release process since HT constraints are ignored at all; as a result, the previous modelling cannot reflect the actual difference in system performance.

On the above premises, the aim of this work is to propose a general model for the TES device which considers the detailed HT process, and can be used to for the dispatch of both SH and LH TES. In our previous work [29], we have proposed a dispatch model for phase-change TES, namely, LH TES, considering HT process. The modelling work in [29] is based on the demonstration project under construction in Northern China and it is more engineering focused due to the limitations of the real devices, in which we assume that the heat conduction medium for the heat storage process always operates at its maximum capacity. In this paper, we removed this assumption, and further develop this model into a general and comprehensive TES model. Here is a summary of this paper's main contributions:

1) A general model for TES device which considers the detailed HT constraints is proposed: this model can be used to describe both SH and LH TES by changing relevant model parameters, and enables a more precise analysis of the flexibility that the TES device can provide for CHP operation;

2) To deal with the complex nonlinear equality constraints introduced by the HT process, we propose an iteration procedure in which each iteration is a linear optimization and the nonlinear constraints are processed outside the iteration by interpolation;

3) The differences between the already widely used $\mathrm{SH}$ TES and the emerging LH TES are compared in terms of provision of extra flexibility for CHP, based on the proposed general model.

This paper begins in Section III by describing the system under analysis and some basic assumptions; Section IV details the general TES model; Section $\mathrm{V}$ provides the basic discussions and the solution method; case studies are presented in Section VI and Section VII gives the conclusions.

\section{SYSTEM DESCRIPTION AND ASSUMPTIONS}

Fig.1 details the structure of the TES device installed in a CHP power plant. Here we use SH TES device as an example to illustrate the structure, and in the following model description section we will present the general model in detail and distinguish the SH TES and LH TES. It needs to be mentioned that this is just one possible structure for TES, and there are several other possible structures [14][15] within a CHP plant.

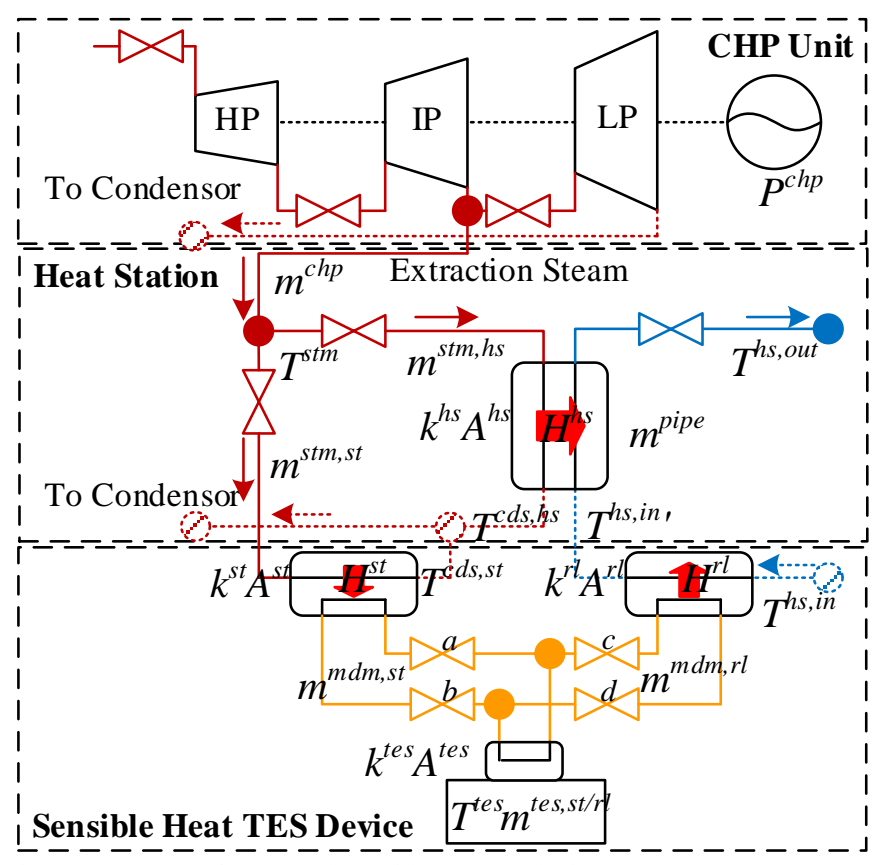

Fig. 1. Structure of a CHP plant with SH TES device

We considers an extraction turbine CHP here. The extraction steam from the intermediate pressure (IP) turbine with mass flow rate $m^{c h p}$ is divided into two flows with $m^{\text {stm,hs }}$ and $m^{\text {stm,st }}$, respectively. The first part with mass flow rate $m^{s t m, h s}$ flows through the heat exchanger in the heat station and is used to heat the pipeline water from $T^{h s, i n}$ to $T^{h s, o u t}$ with the transferred heat $H^{h s}$. The second part with $m^{\text {stm,st }}$ flows through the heat storage heat exchanger and transfers heat $H^{s t}$ to the heat conduction medium if opens valves $a$ and $b$, and $H^{s t}$ will be further transferred into the TES device. The TES device can transfer heat $H^{r l}$ to the heat conduction medium by opening valves $c$ and $d$, and $H^{r l}$ will be further transferred into the pipeline to heat the water from $T^{h s, i n}$ to $T^{h s, i n}$.

Here we firstly provide inputs and assumptions for the 
successive modelling.

1) The temperature and pressure of the extraction steam are the design parameters of the turbine, which are constant values, and this makes the phase-change temperature of the extraction steam also known value;

2) All the HT areas $\left(A^{h s}, A^{s t}, A^{r l}\right.$ and $\left.A^{\text {tes }}\right)$ are design parameters of the heat exchangers. Besides, according to [30], since the fluid materials are given, and the mass flow rates in the heat exchangers are relatively small in this study, the HT coefficients $\left(k^{h s}, k^{s t}, k^{r l}\right.$ and $\left.k^{\text {tes }}\right)$ can also be regarded as constant;

3) The district heating system works with a constant flow and variable temperature mode [21][23][25], namely, $m^{\text {pipe }}$ is fixed and known, and $T^{\text {hs, out }}$ can be adjusted. According to the real operation scheme in Northern China, $T^{h s, \text { out }}$ is adjusted based on the average environment temperature, and it will keep unchanged during a short period of time like a few days [23][30]. This paper considers the daily operation of the TES, thus $T^{\text {hs,out }}$ can also be regarded as constant.

4) The temperature of the TES material is unified, namely, for both the heat storage and release process, after the fluid with temperature $T^{\text {tes,out }}$ flows out of the heat exchanger, it will mix with the rest of the TES material immediately, and the TES material reaches a new $T^{\text {tes }}$ (for the LH TES it will just keep its phase-change temperature).

\section{A GENERAL MODEL FOR THE TES}

In Fig.1, there are two types of HT processes for the TES device. The first type is the HT between the extraction steam and the district heating water or the heat conduction medium, which involves the steam's phase-change process and thus is very complex. The other type is the HT between two liquid fluids, for example the HT between the heat conduction medium and the TES material or the district heating water, which is simpler than the previous one since there is no phasechange process.

The steam's HT process can be described using the threestage HT model proposed in [30]. Reference [30] divides the steam's HT process into three sub processes according to the change of the steam's physical properties during this process, namely from gas state to gas-liquid state and finally to liquid state, and proposes an iteration method to get the needed steam mass flow rate from a given heat demand. It is illustrated that if the cold side fluid's mass flow rate and inlet/outlet temperature are known, then there will be a corresponding relationship between the given heat demand and the hot side steam mass flow rate.

While the common HT between two liquid fluids can be described using the entransy dissipation-based thermal resistance (EDTR) theory [31][32], whose key formulas are recalled below.

\section{A. Model for Heat Storage Process}

Fig. 2 presents the structure of heat storage process.

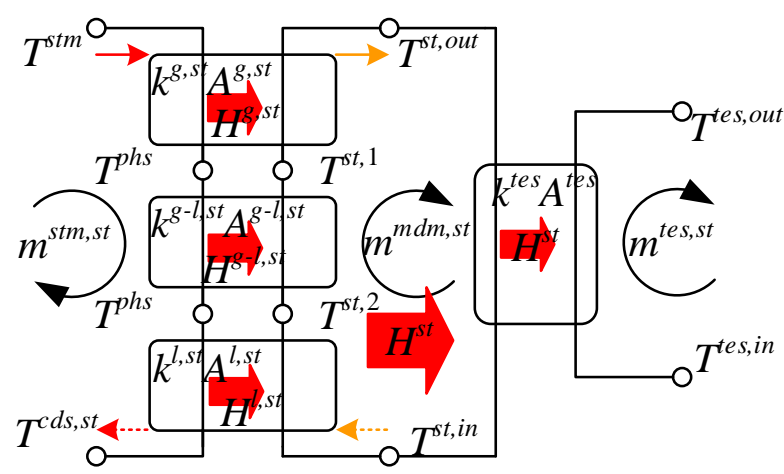

Fig. 2. Structure of the heat storage process

In Fig.2, the left half is the three-stage HT description between the steam and the heat conduction medium, whose three sub processes can be described using the general HT equations

$$
\begin{aligned}
& H^{g, s t}=k^{g, s t} A^{g, s t} \frac{\left(T^{s t m}-T^{s t, \text { out }}\right)-\left(T^{p h s}-T^{s t, 1}\right)}{\ln \left(\left(T^{s t m}-T^{s t, \text { out }}\right) /\left(T^{p h s}-T^{s t, 1}\right)\right)} \\
& H^{g-l, s t}=k^{g-l, s t} A^{g-l, s t} \frac{\left(T^{p h s}-T^{s t, 1}\right)-\left(T^{p h s}-T^{s t, 2}\right)}{\ln \left(\left(T^{p h s}-T^{s t, 1}\right) /\left(T^{p h s}-T^{s t, 2}\right)\right)} \\
& H^{l, s t}=k^{l, s t} A^{l, s t} \frac{\left(T^{p h s}-T^{s t, 2}\right)-\left(T^{c d s, s t}-T^{s t, \text { in }}\right)}{\ln \left(\left(T^{p h s}-T^{s t, 2}\right) /\left(T^{c d s, s t}-T^{s t, \text { in }}\right)\right)}
\end{aligned}
$$

The HT between the heat conduction medium and the TES material can be described based on the EDTR theory

$$
\begin{aligned}
& H^{s t}=\frac{T^{\text {st, out }}-T^{\text {tes }}}{R^{\text {en,tes,st }}} \\
& R^{e n, t e s, s t}=\frac{m^{t e s, s t} c^{t e s} e^{\frac{k^{t e s} A^{t e s s}}{m^{m d m, s t} c^{m d m}}}-m^{m d m, s t} c^{m d m} e^{\frac{k^{t e s} A^{t e s s}}{m^{t e s t} c^{t e s}}}}{m^{m d m, s t} c^{m d m} m^{t e s, s t} c^{t e s}\left(e^{\frac{k^{t e s} A^{t e s}}{m^{m d m, s t} c^{m d m}}}-e^{\frac{k^{t e s} A^{t e s s} c^{t e s}}{m^{t e s}}}\right)}
\end{aligned}
$$

As illustrated in (2), the heat storage process is closely related to the TES material temperature $T^{\text {tes }}$. For the SH TES, since $T^{\text {tes }}$ actually reflects the total thermal energy stored, then there should be different heat storage power with different thermal storage level. Besides, the heat storage power is also related to $T^{\text {st,out }}, m^{m d m, s t}$ and $m^{\text {tes,st }}$.

The flexibility provided by the TES device depends to some extent on the heat storage power limit. This limit is related to two factors: the first one is the temperature difference constraints of the steam's HT, namely the hot fluid should have a higher temperature than the cold fluid, and the other one is the constraints from these HT equations.

From the first factor, we can get (3) by combining the energy equations of the HT and the temperature difference constraints

$$
H^{s t} \leq \frac{T^{p h s}-T^{t e s}}{R^{\text {en,tes, st }}-\frac{c^{s t m}\left(T^{s t m}-T^{p h s}\right)}{\left(c^{s t m}\left(T^{s t m}-T^{p h s}\right)+r\right) m^{m d m, s t} c^{m d m}}}
$$

As presented in (3), for a given $T^{t e s}$, the varying of $m^{m d m, s t}$ and $m^{\text {tesst }}$ will influence the heat storage power limit, and the trend is easy to be demonstrated as shown in Fig. 3 that the 


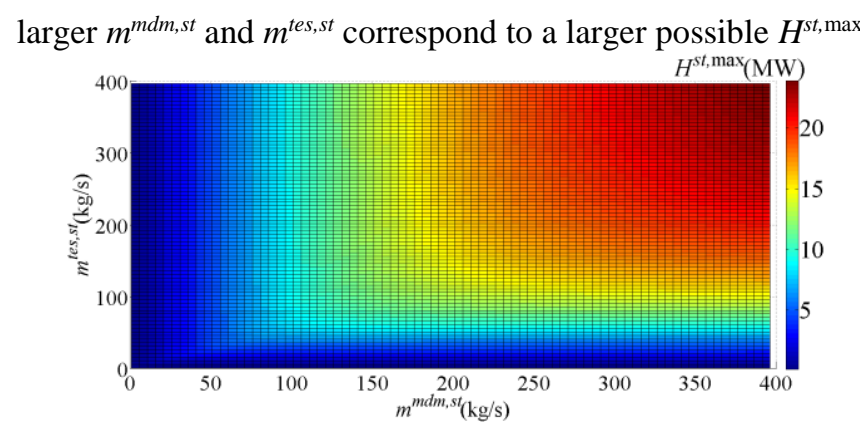

Fig. 3. Trend of $H^{s t, \max }$ by (3) when $m^{m d m, s t}$ and $m^{t e s, s t}$ vary $\left(T^{\text {tes }}=90^{\circ} \mathrm{C}\right)$

The second factor's influence on $H^{s, \max }$ is more complicated compared with the first one, as it needs to be analyzed through the detailed HT equations, which can be realized by the iteration method proposed in [30], this analysis will be detailed in the next section.

\section{B. Model for Heat Release Process}

Fig. 4 shows the structure of heat release process.

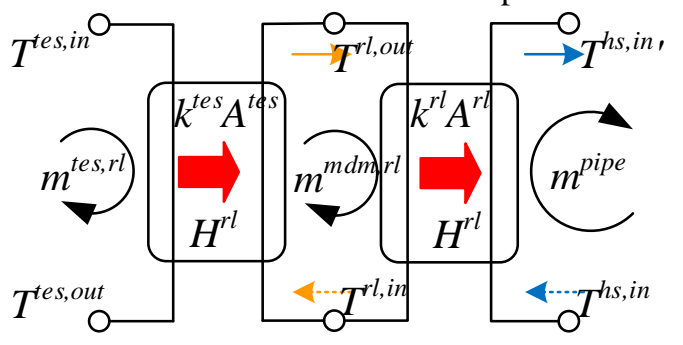

Fig. 4. Structure of the heat release process

The heat release process can be described as

$$
\begin{aligned}
& H^{r l}=\frac{T^{\text {tes }}-T^{h s, i n}}{R^{e n, r l}+R^{e n, t e s, r l}-1 / m^{m d m, r l} c^{m d m}} \\
& R^{e n, t e s, r l}=\frac{m^{m d m, r l} c^{m d m} e^{\frac{k^{\text {tes }} A^{\text {tes }}}{m^{\text {tes }, r l} c^{\text {tes }}}}-m^{\text {tes,rl }, c^{\text {tes }}} e^{\frac{k^{\text {tes }} A^{\text {tes }}}{m^{m d m, r l} c^{m d m}}}}{m^{\text {tes, } r l} c^{\text {tes }} m^{m d m, r l} c^{m d m}\left(e^{\frac{k^{\text {tes }} A^{\text {tes }}}{m^{\text {tes, }, r l} c^{\text {tes }}}}-e^{\frac{k^{\text {tes }} A^{\text {tes }}}{m^{m d m, r l} c^{m d m}}}\right)} \\
& R^{e n, r l}=\frac{m^{\text {pipe }} c^{\text {water }} e^{\frac{k^{r l} A^{r l}}{m^{m d m, r l} c^{m d m}}}-m^{m d m, r l} c^{m d m} e^{\frac{k^{r l} A^{r l}}{m^{p i p e} c^{\text {water }}}}}{m^{m d m, r l} c^{m d m} m^{\text {pipe }} c^{\text {water }}\left(\frac{k^{r l} A^{r l}}{e^{m^{m d m, r l} c^{m d m}}}-\frac{k^{r l} A^{r l}}{m^{p i p e} c^{\text {water }}}\right)}
\end{aligned}
$$

$m^{p i p e}$ keeps unchanged for operation, thus, the denominator of the HT equation in (4) is a function of $m^{m d m, r l}$ and $m^{t e s, r l}$, the relationship between the denominator and the two mass flow rates is shown in Fig.5 (only shows values $\leq 5$ ). From Fig. 5 we can see that larger $m^{m d m, r l}$ and $m^{\text {tes,rl }}$ correspond to a smaller denominator. Thus by substituting $m^{m d m, r l, \max }$ and $m^{\text {tes, } r l, \max }$ into (4), then $H^{r l \text { max }}$, which also relates to the flexibility the TES can provide, will only depends on the difference of $T^{\text {tes }}$ and $T^{h s, i n}$.

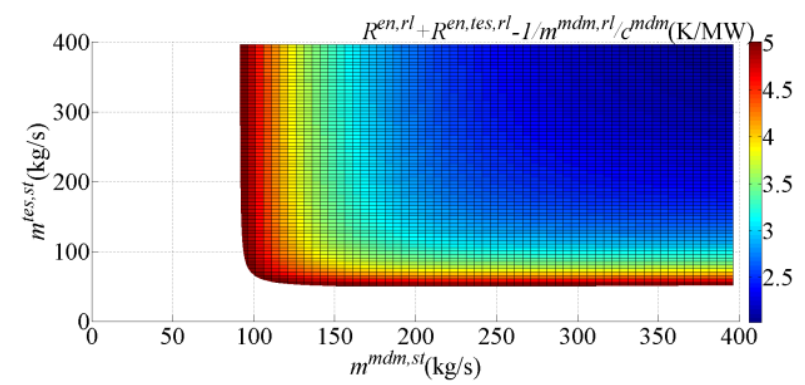

Fig. 5. Trend of $R^{e n, r l}+R^{e n, t e s, r l}-1 / m^{m d m, r l} c^{m d m}$ by (4) when $m^{m d m, r l}$ and $m^{t e s, r l}$ vary

\section{Thermal Energy Balance}

In previous studies, the difference between SH TES model and LH TES model mainly lies in the thermal energy balance equations, where the $\mathrm{SH}$ model uses the $T^{\text {tes }}$ as the decision variable, while $\Phi^{\text {tes }}$ for LH, so we have (5) for SH TES and (6) for LH TES.

$$
\begin{gathered}
c^{t e s} V^{t e s} d^{t e s}\left(T_{t+1}^{t e s}-T_{t}^{t e s}\right)=\left(H_{t}^{s t}-H_{t}^{r l}-H_{t}^{l s}\right) \Delta t \\
\Phi_{t+1}^{t e s}-\Phi_{t}^{t e s}=\left(H_{t}^{s t}-H_{t}^{r l}-H_{t}^{l s}\right) \Delta t
\end{gathered}
$$

where $H_{t}^{s s}$ can be calculated as

$$
H_{t}^{l s}=\left(T_{t}^{t e s}-T_{t}^{e n v}\right) / R^{t e s}
$$

\section{JOINT DISPATCH MODEL FOR CHP WITH TES}

\section{A. Dispatch Model Description}

For the general TES model introduced, the main difficulty for the optimization is the nonlinear constraints corresponding to the heat storage/release HT process and the direct heating HT process.

By observing these HT equations, for the heat storage process and direct heating process, the nonlinearity mainly lies in the equations that describe the relationship between the steam mass flow rate and the heat power transferred; thus, if we know the heat-mass ratio $\rho^{h-m, h s}$ and $\rho^{h-m, s t}$ which satisfy (8) in advance, then this nonlinearity can be removed.

$$
\begin{aligned}
& H_{t}^{h s}=\rho_{t}^{h-m, h s} \cdot m_{t}^{s t m, h s} \\
& H_{t}^{s t}=\rho_{t}^{h-m, s t} \cdot m_{t}^{s t m, s t}
\end{aligned}
$$

As for the heat release process, $H^{r l \text { max }}$ depends on $\left(T^{t e s}\right.$ $T^{h s, i n}$ ), since $T^{h s, i n}$ is actually known with given heat load and given heat station output temperature. Thus, if we can estimate $T^{t e s}$, then we also have an estimate for $H^{r l, \max }$, so that the nonlinearity in the heat release constraints can also be removed, as shown in (9)

$$
0 \leq H_{t}^{r l} \leq H_{t}^{r l, \max }
$$

Besides, there are power and mass flow limits for the heat storage process

$$
\begin{aligned}
& H_{t}^{s t} \leq H_{t}^{s t, \max } \\
& m_{t}^{s t m, s t} \leq m_{t}^{s t m, s t, \text { max }}
\end{aligned}
$$

where $H_{t}^{t, \text { t,max }}$ and $m_{t}^{\text {stm,s,t,max }}$ are also related to the detailed HT modelling, and their acquisition together with the acquisition of $\rho^{h-m, h s}, \rho^{h-m, s t}$ and $H^{r l, \max }$ will be detailed in the following section. 
The capacity constraints for the TES device are:

$$
\begin{aligned}
& \mathrm{SH}: T^{t e s, \min } \leq T_{t}^{t e s} \leq T^{t e s, \max }, T_{0}^{t e s}=T_{N}^{t e s} \\
& \mathrm{LH}: \Phi^{t e s, \min } \leq \Phi_{t}^{t e s} \leq \Phi^{t e s, \max }, \Phi_{0}^{t e s}=\Phi_{N}^{t e s}
\end{aligned}
$$

The heat demand and steam generation constraints are:

$$
\begin{aligned}
& H_{t}^{h s}+H_{t}^{r l}=H_{t}^{h l} \\
& T_{t}^{h s, i n s}=T_{t}^{h s, i n}+H_{t}^{r l} / m^{\text {pipe }} c^{\text {water }} \\
& m_{t}^{\text {chp }}=m_{t}^{\text {stm,hs }}+m_{t}^{\text {stm,st }}
\end{aligned}
$$

Other constraints include generator constraints (13), electric balance constraint (14), as well as transmission constraints of power network (15) [30].

$$
\begin{gathered}
\left\{\begin{array}{l}
P_{i, t}^{c h p}=\sum_{k \in S_{i}^{c v, c h p}} \eta_{i, k, t} P_{i, k}^{c h p} \\
m_{i, t}^{c h p}=\sum_{k \in S_{i}^{c v, c h p}} \eta_{i, k, t} m_{i, k}^{c h p}, \forall i \in S^{c h p}, \forall t \\
\sum_{k \in S_{i}^{c v, c h p}} \eta_{i, k, t}=1,0 \leq \eta_{i, k, t} \leq 1, \forall k \in S_{i}^{c v, c h p} \\
P_{i}^{t p, \min } \leq P_{i, t}^{t p} \leq P_{i}^{t p, \max }, \forall i \in S^{t p}, \forall t \\
0 \leq P_{i, t}^{w p} \leq P_{i, t}^{w p, \max }, \forall i \in S^{w p}, \forall t \\
-D_{i}^{t p} \leq \frac{P_{i, t+1}^{t p}-P_{i, t}^{t p} \leq U_{i}^{t p}, \forall i \in S^{t p}, \forall t}{\Delta t} \\
-D_{i}^{c h p} \leq \frac{P_{i, t+1}^{c h p}-P_{i, t}^{c h p}}{\Delta t} \leq U_{i}^{c h p}, \forall i \in S^{c h p}, \forall t \\
\sum_{i \in S^{t p}} P_{i, t}^{t p}+\sum_{i \in S^{c h p}} P_{i, t}^{c h p}+\sum_{i \in S^{w p}} P_{i, t}^{w p}=\sum_{i \in S^{e l}} P_{i, t}^{e l}, \forall t \\
-P_{i}^{b r c h, \max } \leq P_{i, t}^{b r c h} \leq P_{i}^{b r c h, \max } \\
P_{i, t}^{\text {brch }}=\sum_{n \in S^{b u s}} F_{i, n} \sum_{j \in S_{n}^{t p}} P_{j, t}^{t p}+\sum_{j \in S_{n}^{c h p}} P_{j, t}^{c h p} P_{j \in S_{n}^{w p}}^{w p}-\sum_{j \in S_{n}^{e l}} P_{j, t}^{e l}
\end{array}\right), \forall i \in S^{b r c h}, \forall t
\end{gathered}
$$

The objective of the optimization is to achieve a minimum coal consumption

$$
\begin{aligned}
& \min \left[\sum_{t=1}^{N}\left(\sum_{i \in S^{t p}} f_{i}^{t p}\left(P_{i, t}^{t p}\right)+\sum_{i \in S^{c h p}} f_{i}^{c h p}\left(P_{i, t}^{c h p}, m_{i, t}^{c h p}\right)\right)\right] \\
& f_{i, t}^{c h p}\left(P_{i, t}^{c h p}, m_{i, t}^{c h p}\right)=\left[\begin{array}{l}
\mu_{i, 1}+\mu_{i, 2} P_{i, t}^{c h p}+\mu_{i, 3} m_{i, t}^{c h p}+\mu_{i, 4}\left(P_{i, t}^{c h p}\right)^{2} \\
+\mu_{i, 5}\left(m_{i, t}^{c h p}\right)^{2}+\mu_{i, 6}\left(P_{i, t}^{c h p}\right)\left(m_{i, t}^{c h p}\right)
\end{array}\right] \cdot \Delta t \\
& f_{i}^{t p}\left(P_{i, t}^{t p}\right)=\left[\lambda_{i, 1}\left(P_{i, t}^{t p}\right)^{2}+\lambda_{i, 2} P_{i, t}^{t p}+\lambda_{i, 3}\right] \cdot \Delta t
\end{aligned}
$$

\section{B. Dealing with the Nonlinear Constraints}

As illustrated by (8) and (9), the nonlinearity of the dispatch problem is removed by introducing $\rho^{h-m, h s}, \rho^{h-m, s t}$ and $H^{r l \text { max }}$; thus, with given $\rho^{h-m, h s}, \rho^{h-m, s t}$ and $H^{r l, m a x}$, this dispatch problem becomes a linear program with quadratic objective, which can be solved by commercial programming packages like IBM CPLEX solver. Therefore, we propose an iteration method as shown in Fig. 6 to deal with these nonlinear constraints.

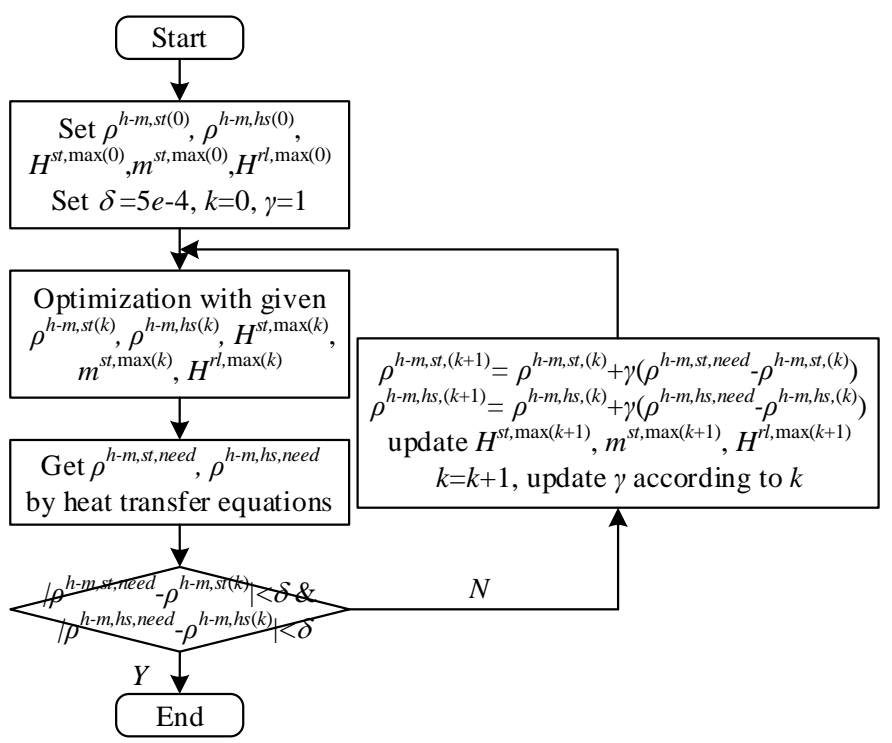

Fig. 6. Iteration flowchart for the optimization

After each optimization, we use optimized $T_{i, t}^{e s}, H_{i, t}^{h s}, T_{i, t}^{h s, i n}, H$ ${ }_{i, t}^{s t}, m_{i, t}^{\text {stm,hs,need }}$ and $m_{i, t}^{\text {stm,st,need }}$ to calculate $\rho_{i, t}^{h-m, h s, \text { need }}$ and $\rho_{i, t}^{h-m, s t, n e d e d}$, then update $\rho_{i, t}^{h-m, h s}$ and $\rho_{i, t}^{h-m, s t}$ for the next iteration. Also, based on $T_{i, t}^{e s}$, the variables $H_{i, t}^{s, \max }, m_{i, t}^{s t, \max }$ and $H_{i, t}^{r, \max }$ should be updated.

It is relatively easier to calculate $\rho_{i, t}^{h-m, h s, n e e d}$ according to the optimized $H_{i, t}^{h s}$ and $T_{i, t}^{h s, i n}$ ' since $m_{i}^{\text {pipe }}$ and $T_{i}^{h s, \text { out }}$ are obtained in the optimization, and we can get $\rho_{i, t}^{h \text {-m,hs,need }}$ by the iteration method proposed in [30].

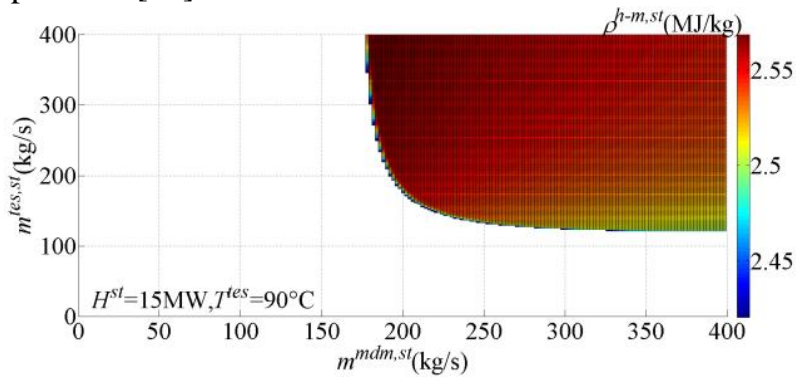

Fig. 7. Trend of $\rho^{h-m, s t}$ when $m^{m d m, s t}$ and $m^{\text {tes,st }}$ vary $\left(T^{\text {tes }}=90^{\circ} \mathrm{C}, H^{s t}=15 \mathrm{MW}\right)$

As for $\rho_{i, t}^{h \text {-mst,needed }}$, it is more complicated due to the fact that both $m_{i, t}^{m d m, s t}$ and $m_{i, t}^{t e s, s t}$ can vary. Thus, for an optimized $H_{i, t}^{s t}$, there might be many combinations of $m_{i, t}^{m d m, s t}$ and $m_{i, t}^{\text {tesst }}$ that can satisfy it. Since the objective is to achieve a minimum coal (16) consumption of the system, then a larger $\rho_{i, t}^{h-m, s t}$ is preferred to satisfy the given heat storage power $H_{i, t}^{\text {st }}$. Fig. 7 gives the trend of $\rho^{h-m, s t}$ when $m^{m d m, s t}$ and $m^{t e s, s t}$ vary $\left(T^{t e s}=90^{\circ} \mathrm{C}, H^{s t}=15 \mathrm{MW}\right)$

It can be seen from Fig.7 that a larger $m^{\text {tes,st }}$ is always corresponding to a larger $\rho^{h-m, s t}$, but the relationship between $m^{m d m, s t}$ and $\rho^{h-m, s t}$ is more complicated. For the extraction steam's HT process, a smaller $T^{\text {st,out }} / T^{\text {st, in }}$ is better for getting a higher $\rho^{h-m, s t}$. A larger $m^{\text {tes,st }}$ means a smaller $R^{e n, t e s, s t}$, so that if we have a known $T^{\text {tes }}$, there can be smaller $T^{\text {stout }}$, and consequently larger $\rho^{h-m, s t}$. Although a larger $m^{m d m, s t}$ also means a smaller $R^{\text {en,tes,st }}$, the difference $T^{\text {st,out }}-T^{\text {st,in }}$ is also related to $m^{m d m, s t}$ : since larger $m^{m d m, s t}$ means smaller $T^{s, o u t}-T^{s t, i n}$, then there might be a larger $T^{\text {st,in }}$, which is not good for the steam's HT process. Therefore, to get the largest $\rho^{h-m, s t}$, we can first fix $m^{\text {tes,st }}$ to $m^{\text {tes,st,max }}$, and then consider the relationship between $m^{m d m, s t}$ and $\rho^{h-m, s t}$. For every given $T^{t e s}$ and $H^{s t}$, we can adjust 
$m^{m d m, s t}$ to get the largest $\rho^{h-m, s t}$. Searching for the largest $\rho^{h-m, s t}$ is another nonlinear optimization problem which is hard to solve. Thus, in this paper we first create a table composed of $T^{t e s}, H^{s t}$ and the corresponding largest $\rho^{h-m, s t}$ using the iteration method proposed in [30]; then, in every iteration, we update the needed $\rho^{h-m, s t}$ by interpolation according to the optimized $T^{t e s}$ and $H^{s t}$ based on the table. An example relationship between $T^{t e s}, H^{s t}$ and $\rho^{h-m, s t}$ is shown in Fig. 8. At the same time, we can also update the $H_{t}^{s, \text { max }}$ and $m_{t}^{\text {stm,st,max }}$ according to the optimized $T^{\text {tes }}$ by the plot shown in Fig.8.

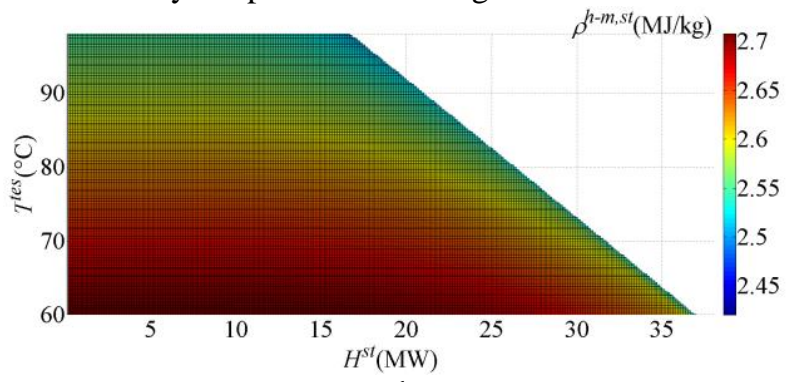

Fig. 8. Relationship to get the needed $\rho^{h-m, s t}$ from $H^{s t}$ and $T^{\text {tes }}$

Fig. 8 illustrates how the TES material temperature $T^{\text {tes }}$ will influence the heat storage process greatly. A larger $T^{\text {tes }}$ means there is more difficulty for the heat storage HT process, thus there will be smaller maximum possible heat storage power $H^{s t \text { max }}$ and smaller $\rho^{h-m, s t}$.

In order to achieve an acceptable convergence and speed up the iteration, a variable updating step $\gamma$ is selected by trial and error, and is presented in Table I. In addition, the iteration threshold used in this study is $\delta=5 \mathrm{e}-4$, and the resolution of the table used for interpolation is $H^{s t}=0.02 \mathrm{MW}$ and $T^{t e s}=0.02^{\circ} \mathrm{C}$.

TABLE I. RELATIONSHIP BETWEEN $\Gamma$ AND $K$

\begin{tabular}{ccccccccccc}
\hline Iteration & {$[0$,} & {$[5$,} & {$[10$,} & {$[20$,} & {$[30$,} & {$[60$,} & {$[150$,} & {$[300$,} & {$[500$,} & {$[700$,} \\
Number $k$ & $5)$ & $10)$ & $20)$ & $30)$ & $60)$ & $150)$ & $300)$ & $500]$ & $700)$ & $\left.k^{\max }\right]$ \\
\hline Updating & 1 & \multirow{2}{*}{0.5} & \multirow{2}{*}{$1 / 4$} & $1 / 8$ & $1 / 16$ & $1 / 32$ & $1 / 64$ & $1 / 128$ & $1 / 256$ & $1 / 512$ \\
Step $\gamma$ & & & & & & & & & \\
\hline
\end{tabular}

\section{From Sensible Heat to Latent Heat}

Except for the thermal energy balance equations, the description of LH TES's heat storage and release model can be to some extent regarded as a special case of SH TES with a constant $T^{\text {tes }}$. For LH TES, since $T^{\text {tes }}$ is constant, the HT equations in (2) and (4) needs to be slightly changed, that is, the TES material's heat capacity rate $\left(m^{\text {tes }} c^{\text {tes }}\right)$ can be regarded as infinite when $T^{t e s}$ is constant [31]. In this case, $R^{e n, t e s, s t}$ and $R^{\text {en,tes, } r l}$ are expressed as

$$
\begin{aligned}
& R^{e n, t e s, s t}=\frac{e^{\frac{k^{\text {tes }} A^{\text {tes }}}{m^{m d m, s t} c^{m d m}}}}{m^{m d m, s t} c^{m d m}\left(e^{\frac{k^{\text {tes }} A^{\text {tes }}}{m^{m d m, s t} c^{m d m}}}-1\right)} \\
& R^{e n, t e s, r l}=\frac{e^{\frac{k^{t e s} A^{t e s}}{m^{m d m, r l} c^{m d m}}}}{m^{m d m, r l} c^{m d m}\left(e^{\frac{k^{t e s} A^{\text {tes }}}{m^{m d m, r l} c^{m d m}}}-1\right)}
\end{aligned}
$$

As for the iteration, since $T^{t e s}$ is fixed, then the table needed for searching $\rho^{h-m, s t}$ will be monodimensional, thus $H_{i, t}^{s, \text { max }}, m_{i, t}^{s, \text { max }}$ and $H_{i, t}^{r, \text { max }}$ will keep unchanged for each iteration, and they don't need to be updated.

It needs to be mentioned that, the general TES model proposed in this study is a case corresponding to the TES structure in Fig. 1. For other TES structures, for example water tank with water stratification which directly connects in the pipelines (there is actually no HT process in such structure), then the proposed model can't be used directly; however, the modelling method and solving procedure can provide reference to their modelling and analysis.

\section{CASE StUdiEs}

\section{A. Test System Description}

We use a 6-bus system [14][21][22][24][29][30] and a real system data from Northern China's Hohhot district for the following case studies.

In the 6-bus system, there are two thermal units (with capacity of $200 \mathrm{MW}$ and $220 \mathrm{MW}$ ), a $220 \mathrm{MW}$ CHP unit and a 200MW wind farm. The SH TES device has a volume of $1500 \mathrm{~m}^{3}$ and its temperature limit is $60^{\circ} \mathrm{C}$ to $98^{\circ} \mathrm{C}$. The peak electric load for the system is $596.5 \mathrm{MW}$, while the peak heat demand is 251.2MW. Fig.9 (a) gives the system structure and Fig.9 (b) the profiles of available wind power, heat demand and total electric load. The parameters of the system are detailed in [33], and $\Delta t=0.25 \mathrm{~h}$ is used in this study.

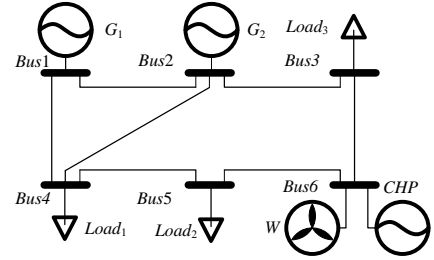

(a)

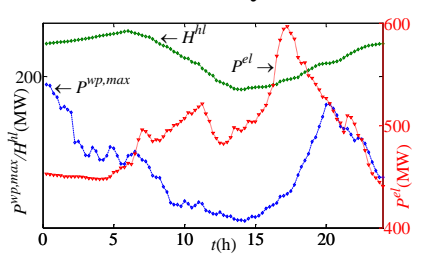

(b)
Fig. 9. (a) 6-bus system structure and (b) profiles of $P^{w p, \max }, H^{h l}$ and $P^{e l}$

In the Hohhot district system, there are 37 buses (only the $220 \mathrm{kV}$ and $500 \mathrm{kV}$ buses are considered) with 12 units (total capacity of $3.6 \mathrm{GW}$ ) and 3 wind farms (total capacity of 0.9GW).

In the following section, we will first discuss the effectiveness of the proposed model and solving method based on the abovementioned two test systems, and then we use the 6-bus system to compare the proposed TES model with the previous model, to illustrate the need for considering the HT constraints. We also compare the SH TES with the LH TES to demonstrate the difference they bring to system performance based on the proposed general model using the 6-bus system.

\section{B. Effectiveness of the Iteration Method and Discussion}

With the iteration parameters presented in Table I, the optimizations of the 6-bus system in the following parts can be solved within about 150 iterations and the solving time is about 15 seconds (all the simulations are performed in a computer with an Intel Core i7 CPU and 8GB memory, and are coded using MATLAB R2012b, the linear optimization in each iteration is solved by IBM CPLEX solver).

Besides, we also test the effectiveness of the proposed iteration method using real power system data from Northern 
China's Hohhot district, where a LH TES demonstration project is under construction. With the iteration parameters presented in Table I, the optimization of this real system can be solved within 800 iterations and the solving time is less than 200 seconds.

Including HT constraints in the modelling makes the dispatch problem a strong nonlinear optimization problem, and to the best of our knowledge, there is actually no "perfect" solution to a nonlinear optimization problem. Therefore, even though the convergence of the proposed iteration method still lacks strict mathematical proof, the simulation results of the abovementioned 6-bus test system and the real system present a satisfactory performance for this nonlinear optimization problem. This shows that the proposed approach is practical and the convergence of the method may be studied in more detail in the future.

\section{Comparison with Previous Models}

In previous studies [13]-[20], $H^{\text {st/rl,max }}$ is modeled to be constant, and in almost all the cases $H^{s, \text { max }}=H^{r l, \text { max }}$. However, as the proposed model suggests, both $H^{s, \text { max }}$ and $H^{r, \text { max }}$ will be influenced by $T^{t e s}$; which reflects the total thermal energy stored in the TES device, and may change greatly during the daily operation. Furthermore, $H^{r l, \max }$ will be influenced by $T^{h s, i n}$ To compare the proposed model with these previous models, based on the results of the detailed model we consider the following two cases to represent the previous model. For c1, $H^{s t, \max }$ and $H^{r, \max }$ are chosen as the minimum value based on the detailed model, so that it is an achievable case and can be used to represent the previous approximated model. For $\mathrm{c} 2$, the average $H^{s t, \max }$ and $H^{r, \text { max }}$ of the detailed model is used. In addition, for both $\mathrm{c} 1$ and $\mathrm{c} 2, \rho^{h-m, h s}$ and $\rho^{h-m, s t}$ use the average value from the detailed model. C4 is the case without TES device but with detailed consideration of the HT process. The descriptions of these four cases is given in Table II. $T^{h s, \text { out }}$ is set to be $90^{\circ} \mathrm{C}$ for all the four cases.

TABLE II. CASE STUDES AND KEY PARAMETERS

\begin{tabular}{ccccc}
\hline Cases & $\rho^{h-m, h s}$ & $\rho^{h-m, s t}$ & $H^{s, \text { max }}$ & $H^{r l, \max }$ \\
\hline $\mathrm{c} 1$ & 2.7046 & 2.5679 & 16.7700 & 4.6330 \\
$\mathrm{c} 2$ & 2.7046 & 2.5679 & 24.0788 & 14.4148 \\
$\mathrm{c} 3$ & time-varying & time-varying & time-varying & time-varying \\
$\mathrm{c} 4$ & time-varying & $\backslash$ & $\backslash$ & $\backslash$ \\
\hline
\end{tabular}

The dispatch results are presented in Table III, while Fig.10 gives the profiles of $P^{w p}$ and $T^{\text {es }}$ and Fig.11 shows $H^{\text {strl }}$ and $H^{s t r l, \max }$ for c1 to $\mathrm{c} 3$ in (a) to (c), and (d) gives the relationship between $T^{\text {tes }}$ and $\rho^{h-m, s t}$ for $\mathrm{c} 3$.

TABLE III. DisPatch RESUlTS

\begin{tabular}{ccccccc}
\hline Cases & $\begin{array}{c}\text { Coal } \\
\text { Use } \\
\text { (TCE) }\end{array}$ & $\begin{array}{c}\text { Wind } \\
\text { Power } \\
\text { Gen.(MWh) }\end{array}$ & $\begin{array}{c}\text { Thermal } \\
\text { Power } \\
\text { Gen. } \\
(\mathrm{MWh})\end{array}$ & $\begin{array}{c}\text { CHP Power } \\
\text { Gen.(MWh) }\end{array}$ & $\begin{array}{c}\text { CHP } \\
\text { Heat } \\
\text { Gen. } \\
(\mathrm{MWh})\end{array}$ & $\begin{array}{c}\text { CHP } \\
\text { Steam } \\
\text { Gen. } \\
\text { (t) }\end{array}$ \\
\hline c1 & 3446.0 & 2015.3 & 6467.4 & 3391.0 & 5311.0 & 7073.2 \\
c2 & 3442.4 & 2028.0 & 6465.3 & 3380.5 & 5310.7 & 7076.0 \\
c3 & 3442.4 & 2028.3 & 6455.3 & 3390.3 & 5311.3 & 7077.1 \\
c4 & 3450.6 & 1998.1 & 6473.0 & 3402.7 & 5307.1 & 7067.1 \\
\hline
\end{tabular}


to guarantee that the $\rho^{h-m, s t}$ will not be too small, both to keep a high efficiency and to reduce the steam required. Further, if not considering the HT process as shown in Fig. 11(a) and (b), the dispatch results will use $H^{s t \text { max }}$ instead of the smaller but more efficient one as in our detailed model. This further illustrated the necessity of considering the HT constraints.

\section{Comparison of Sensible Heat TES with Latent Heat TES}

To compare SH TES with LH TES, we choose the same TES capacity and starting thermal energy level (corresponding to $90^{\circ} \mathrm{C}$ of the $\mathrm{SH}$ ), and the phase-change material temperature is chosen as $90^{\circ} \mathrm{C}$ for LH TES. The dispatch results are given in Table IV.

TABLE IV. DISPATCH RESULTS FOR LH AND SH

\begin{tabular}{ccccccc}
\hline Cases & $\begin{array}{c}\text { Coal } \\
\text { Use } \\
(\mathrm{TCE})\end{array}$ & $\begin{array}{c}\text { Wind } \\
\text { Power } \\
\text { Gen.(MWh) }\end{array}$ & $\begin{array}{c}\text { Thermal } \\
\text { Power } \\
\text { Gen. } \\
(\mathrm{MWh})\end{array}$ & $\begin{array}{c}\text { CHP Power } \\
\text { Gen.(MWh) }\end{array}$ & $\begin{array}{c}\text { CHP } \\
\text { Heat } \\
\text { Gen. } \\
(\mathrm{MWh})\end{array}$ & $\begin{array}{c}\text { CHP } \\
\text { Steam } \\
\text { Gen. } \\
(\mathrm{t})\end{array}$ \\
\hline LH & 3442.2 & 2029.0 & 6453.1 & 3391.7 & 5311.6 & 7078.8 \\
SH & 3442.4 & 2028.3 & 6455.3 & 3390.3 & 5311.3 & 7077.1 \\
\hline
\end{tabular}

From Table IV we can learn that LH TES performs better than the SH TES: there is more wind power integrated and less coal consumed. This better performance is mainly due to the fact that there is a more balanced heat storage and heat release process for LH TES. As shown in Fig. 12(a), $H^{s t, \max }$ for LH keeps unchanged and $H^{r l \text { max }}$ almost unchanged, and the values are approximately equal, while there is a large variation for $H^{s t / l l, m a x}$ of SH due to the variation of the $T^{t e s}$. Besides, since the $T^{\text {tes }}$ of LH TES keeps unchanged for the whole day and is higher than SH TES for most of the time, there is more heat loss for the LH TES as presented in Fig.12 (b) and more steam and heat generated as shown in Table IV. However, the performance differences between SH and LH TES are rather small in this study case. This is mainly because the starting thermal energy level we choose is high (corresponds to $90^{\circ} \mathrm{C}$ of the SH TES material). Since the wind curtailment happens mainly during the first several hours of the day as shown in Fig 10(a), during this period, both of the SH and LH TES devices have enough energy to be released for wind integration. Because there are almost 8 hours for TES device to release this starting thermal energy, therefore the difference between the heat release processes will not have a distinct influence on the wind accommodation results.
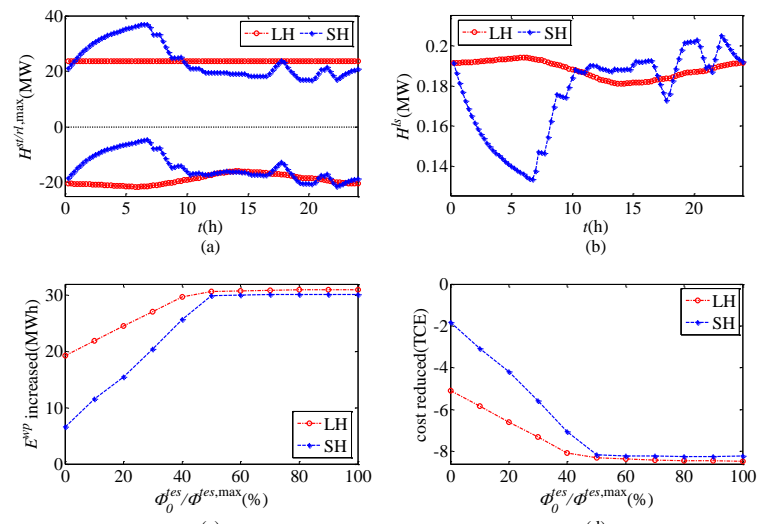

Fig. 12. Profiles of (a) $H^{s t / r l, m a x}$, (b) $H^{l s}$ and (c) increased wind consumption (d) reduced coal use with different starting thermal energy level

TABLE V. INCREASED WIND CONSUMPTION AND REDUCED COAL USE WITH DIFFERENT STARTING THERMAL ENERGY LEVEL FOR LH AND SH

\begin{tabular}{|c|c|c|c|c|c|c|c|c|c|c|c|c|}
\hline \multicolumn{2}{|c|}{$\Phi_{0}^{t e s} / \Phi^{t e s, \max }(\%)$} & 0 & 10 & 20 & 30 & 40 & 50 & 60 & 70 & 80 & 90 & 100 \\
\hline \multirow{2}{*}{$\begin{array}{c}E^{w p} \\
(\mathrm{MWh})\end{array}$} & LH & 19.26 & 21.89 & 27.5 & 21. & 29.69 & 30.67 & 30.79 & 30.88 & 30.95 & 30.9 & 30.99 \\
\hline & $\mathrm{SH}$ & 6.61 & 11.61 & 15.43 & 20.43 & 25.75 & 29.94 & 30.05 & 30.11 & 30.1 & 30. & 30.10 \\
\hline \multirow{2}{*}{$\begin{array}{c}\text { cost } \\
(\mathrm{TCE})\end{array}$} & LH & -5.09 & -5.84 & -6.60 & -7.32 & -8.06 & -8.30 & -8.37 & -8.41 & -8.44 & 8.4 & -8.47 \\
\hline & $\mathrm{SH}$ & -1.83 & -3.09 & -4.19 & -5.58 & -7.05 & -8.15 & -8.21 & -8.22 & -8.24 & -8.25 & -8.22 \\
\hline
\end{tabular}

The benefits of a more balanced heat storage and release processes can be demonstrated more obviously with Fig.12(c) and (d) and Table V. If the starting thermal energy level varies, we can learn from Fig.12(c) and (d) and Table V that there will be large difference in both the increased wind consumption and reduced coal use compared with the case without TES. For example, the increased wind energy accommodation by LH TES can be twice more than that of $\mathrm{SH}$ TES when there is no starting thermal energy (6.61MWh more wind energy accommodated for $\mathrm{SH}$, while 19.26MWh for $\mathrm{LH}$ ), and so is the reduced coal use $1.83 \mathrm{TCE}$ reduced for $\mathrm{SH}$, 5.09TCE for LH). This huge performance difference between LH and SH TES will become smaller as the starting thermal energy level increases.

Moreover, it should be noticed that the HT parameters used for $\mathrm{LH}$ are the same as $\mathrm{SH}$ for the convenience of comparison in this study. However, in reality the HT coefficients are related to the material's physical properties, so that the HT parameters would not be the same for $\mathrm{LH}$ and $\mathrm{SH}$ in a real system.

\section{CONCLUSIONS}

In this study, we proposed a general model for thermal energy storage (TES) with detailed consideration of the heat transfer (HT) constraints. This general model can be used to model both the sensible heat (SH) TES and latent heat (LH) TES by using different parameters. An iteration method is given to solve the dispatch problem with these complex nonlinear HT constraints, and interpolation is used to deal with the strong nonlinearity. The case studies demonstrate the effectiveness of the proposed model and solving method. It is also illustrated how considering the HT process is essential to realistically quantify and make full use of the flexibility that the TES can provide. The differences between $\mathrm{LH}$ and $\mathrm{SH}$ TES are also analyzed, and the results show that LH can provide more flexibility than $\mathrm{SH}$, especially when the starting thermal energy level is low.

Flexibility provided by TES devices will be highly influenced by the HT processes, as demonstrated here. Therefore, future studies will aim to include HT constraints into other thermal flexibility enhancement options such as using the TES capacity of the district heating network, electric boilers, flexible heat consumption, etc. 


\section{REFERENCES}

[1] International Energy Agency, The Power of Transformation: Wind, Sun and the Economics of Flexible Power Systems, 2014.

[2] J. Kiviluoma, S. Heinen, H. Qazi, et al., "Harnessing Flexibility from Hot and Cold," IEEE Power and Energy Magazine, vol. 15, pp. 25-33, 2017.

[3] E. Dall'Anese, P. Mancarella and A. Monti, "Unlocking Flexibility: Integrated Optimization and Control of Multienergy Systems," IEEE Power and Energy Magazine, vol. 15, pp. 43-52, 2017.

[4] C. Kang, X. Chen, Q. Xu, et al., "Balance of Power: Toward a More Environmentally Friendly, Efficient, and Effective Integration of Energy Systems in China," IEEE Power and Energy Magazine, v11, pp. 56-64, 2013.

[5] H. Lund, "Electric grid stability and the design of sustainable energy systems," International Journal of Sustainable Energy, vol. 24, no. 1, pp. 45-54, 2005.

[6] National Energy Administration. "China's wind power industry development in 2016", 2017 [Online]. Available: http://www.nea.gov.cn/2017-01/26/c_136014615.htm

[7] S. Kuravi, J. Trahan, D. Y. Goswami, et al., "Thermal energy storage technologies and systems for concentrating solar power plants," Progress in Energy and Combustion Science, vol. 39, pp. 285-319, 2013.

[8] H. Lund and W. W. Clark, "Management of fluctuations in wind power and CHP comparing two possible Danish strategies," Energy, vol. 27, pp. 471-483, 2002.

[9] H. Lund and E. Munster, "Modelling of energy systems with a high percentage of CHP and wind power," Renewable Energy, vol. 28, pp. 2179-2193, 2003.

[10] H. Lund, "Large-scale integration of wind power into different energy systems," Energy, vol. 30, pp. 2402-2412, 2005.

[11] H. Lund and A. N. Andersen, "Optimal designs of small CHP plants in a market with fluctuating electricity prices," Energy Conversion and Management, vol. 46, pp. 893-904, 2005.

[12] H. Lund, A. N. Andersen, P. A. Østergaard, B. V. Mathiesen, and D. Connolly, "From electricity smart grids to smart energy systems - A market operation based approach and understanding," Energy, vol. 42, pp. 96-102, 2012.

[13] T. Capuder and P. Mancarella, "Techno-economic and environmental modelling and optimization of flexible distributed multi-generation options," Energy, vol. 71, pp. 516-533, 2014.

[14] X. Chen, C. Kang, M. O'Malley, Q. Xia, J. Bai, C. Liu, R. Sun, W. Wang, and $\mathrm{H}$. Li, "Increasing the Flexibility of Combined Heat and Power for Wind Power Integration in China: Modeling and Implications," IEEE Trans. on Power Systems, vol. 30, pp. 1848-1857, 2015.

[15] K. Hu, L. Chen, Q. Chen, et al, "Phase-change heat storage installation in combined heat and power plants for integration of renewable energy sources into power system," Energy, vol. 124, pp. 640-651, 2017.

[16] R. Yuan, J. Ye, J. Lei, and T. Li, "Integrated Combined Heat and Power System Dispatch Considering Electrical and Thermal Energy Storage," Energies, vol. 9, 2016.

[17] H. Wang, W. Yin, E. Abdollahi, R. Lahdelma, and W. Jiao, "Modelling and optimization of CHP based district heating system with renewable energy production and energy storage," Applied Energy, vol. 159, pp. 401-421, 2015.

[18] T. Fang and R. Lahdelma, "Optimization of combined heat and power production with heat storage based on sliding time window method," Applied Energy, vol. 162, pp. 723-732, 2016-01-15 2016.

[19] J. Li, J. Fang, Q. Zeng, and Z. Chen, "Optimal operation of the integrated electrical and heating systems to accommodate the intermittent renewable sources," Applied Energy, vol. 167, pp. 244-254, 2016.

[20] Y. Yang, K. Wu, X. Yan, J. Gao, and H. Long, "The large-scale wind power integration using the integrated heating load and heating storage control," in 2015 IEEE Eindhoven PowerTech, Eindhoven, Netherlands, 2015, pp. 1-6.

[21] Z. Li, W. Wu, M. Shahidehpour, J. Wang, and B. Zhang, "Combined Heat and Power Dispatch Considering Pipeline Energy Storage of District Heating Network," IEEE Transactions on Sustainable Energy, vol. 7, pp. 12-22, 2016.
[22] Z. Li, W. Wu, J. Wang, B. Zhang, and T. Zheng, "TransmissionConstrained Unit Commitment Considering Combined Electricity and District Heating Networks," IEEE Trans. on Sustainable Energy, vol. 7, pp. 480-492, 2016.

[23] Y. Dai, L. Chen, Y. Min, et al., "Modeling and analysis of electrical heating system based on entransy dissipation-based thermal resistance theory," IEEE Power and Energy Society General Meeting (PESGM), 2016, pp. 1-5.

[24] Y. Dai, L. Chen, Y. Min, et al., "Active and Passive Thermal Energy Storage in Combined Heat and Power Plants to Promote Wind Power Accommodation," Journal of Energy Engineering, vol. 143, 2017.

[25] Z. Pan, Q. Guo and H. Sun, "Feasible region method based integrated heat and electricity dispatch considering building thermal inertia," Applied Energy, vol. 192, pp. 395-407, 2017.

[26] A. Rong, R. Lahdelma and P. B. Luh, "Lagrangian relaxation based algorithm for trigeneration planning with storages," European Journal of Operational Research, vol. 188, pp. 240-257, 2008.

[27] E. A. M. Ceseña, T. Capuder and P. Mancarella, "Flexible Distributed Multienergy Generation System Expansion Planning Under Uncertainty," IEEE Trans. on Smart Grid, vol. 7, pp. 348-357, 2016.

[28] IEA-ETSAP and IRENA, "Thermal Energy Storage Technology Brief," $2013 . \quad$ [Online]. Available: https://www.irena.org/DocumentDownloads/Publications/IRENAETSAP\%20Tech\%20Brief\%20E17\%20Thermal\%20Energy\%20Storage .pdf

[29] Y. Dai, L. Chen, Y. Min, P. Mancarella, et al. "Integrated Dispatch Model for Combined Heat and Power Plant with Phase-change Thermal Energy Storage Considering Heat Transfer Process," IEEE Transactions on Sustainable Energy, vol. PP, p. 1-1, 2017..

[30] Y. Dai, L. Chen, Y. Min, et al., "Dispatch Model of Combined Heat and Power Plant Considering Heat Transfer Process," IEEE Transactions on Sustainable Energy, vol. 8, pp. 1225-1236, 2017.

[31] Q. Chen, "Entransy dissipation-based thermal resistance method for heat exchanger performance design and optimization," International Journal of Heat and Mass Transfer, vol. 60, pp. 156-162, 2013.

[32] Q. Chen, R. Fu and Y. Xu, "Electrical circuit analogy for heat transfer analysis and optimization in heat exchanger networks," Applied Energy, vol. 139, pp. 81-92, 2015

[33] "Test System Data for A General Model for Thermal Energy Storage in CHP Dispatch Considering Heat Transfer Constraints ". 2017. Available: https://pan.baidu.com/s/1pKRJmYn

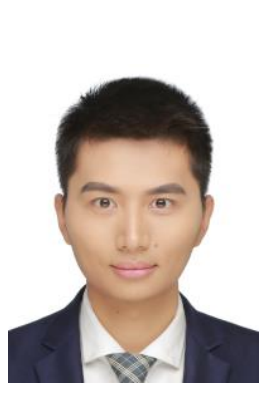

\section{BIOGRAPHIES}

Yuanhang Dai (S'15) was born in China in 1990. $\mathrm{He}$ received the B.Sc. degree in electrical engineering from Tsinghua University, Beijing, China, in 2013, where he is currently pursuing the $\mathrm{Ph} . \mathrm{D}$. degree. His research interests are in modelling and analysis of the integrated electrical-thermal system.

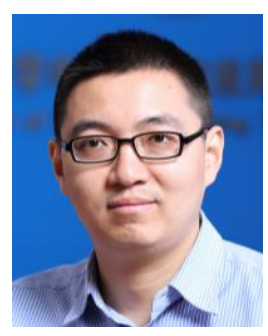

Lei Chen (M'09) was born in China in 1982. He received the B.Sc. degree and the Ph.D. degree in electrical engineering from Tsinghua University, Beijing, China, in 2003 and 2008, respectively. He is now an Associate Professor with the Department of Electrical Engineering. His research interests are in dynamic analysis and control of power systems.

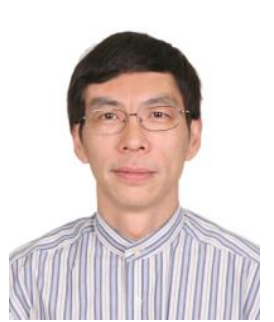

Yong Min was born in China in 1963. He received the B.Sc. degree and the Ph.D. degree in electrical engineering from Tsinghua University, Beijing, China, in 1984 and 1990, respectively. $\mathrm{He}$ is currently a Professor with the Department of Electrical Engineering, Tsinghua University. His research interests are mainly in power system 
stability analysis, renewable energy source integration and smart grid technologies

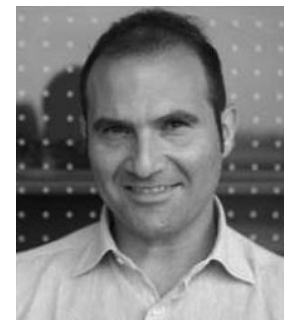

Pierluigi Mancarella (M'08-SM'14) received the $\mathrm{MSc}$ and $\mathrm{PhD}$ degrees in electrical energy systems from the Politecnico di Torino, Torino, Italy, in 2002 and 2006, respectively. He is currently Chair Professor of Electrical Power Systems at The University of Melbourne, Australia, and Professor of Smart Energy Systems at The University of Manchester, UK. His research interests include multi-energy systems, power system integration of low carbon technologies, network planning under uncertainty, and risk and resilience of smart grids. Pierluigi is an Editor of the IEEE Transactions on Smart Grid, an Associate Editor of the IEEE Systems Journal, and an IEEE PES Distinguished Lecturer.

Qun Chen (M'16) was born in China in 1981. He is currently a Professor with the Department of Engineering Mechanics, Tsinghua University.

Junhong Hao is a Post-Doc Researcher with the Department of Electrical Engineering, Tsinghua University.

Kang Hu is a Ph.D. student in the Department of Engineering Mechanics, Tsinghua University.

Fei Xu is an Associate Professor with the Department of Electrical Engineering, Tsinghua University. 